\title{
Breathing Movements of the Chest and Abdominal Wall in Healthy Subjects
}

\author{
Hideo Kaneko PT PhD and Jun Horie PT PhD
}

\begin{abstract}
BACKGROUND: Physical assessment of breathing is an important component of physical therapy evaluations. However, there are no standardized reference values of breathing movements available for use in clinical practice. The purpose of this study was to determine the 3-dimensional distances of observational points on the thorax and abdomen during breathing in healthy subjects and to assess the effects of age, posture, and sex on breathing movements. METHODS: We studied the 3-dimensional breathing movement distances of the thorax and abdomen in $\mathbf{1 0 0}$ healthy subjects (50 males, 50 females). Breathing movements were measured with a 3-dimensional motion system during quiet and deep breathing with subjects in supine and sitting positions. Thirteen reflective markers were placed on the upper (the clavicles, 3rd ribs, and sternal angle) and lower thorax (the 8th ribs, 10th ribs, and xiphoid process) and the abdomen (upper abdomen and lateral abdomen). Range of movement in both breathing conditions was measured as the 3-dimensional distance at half respiratory cycle. Respiratory rates were calculated based on the breathing movements analyzed. One-way analysis of variance, $t$ tests, and multiple regression were used for statistical analysis. RESULTS: The average marker distances for the thorax and abdomen during quiet breathing were less than one third of those during deep breathing. Upper thoracic movement was significantly decreased with age. There was less abdominal movement in females than in males, except during quiet breathing in the supine position. The distances between the thoracic markers were greater and those of the abdomen were less during quiet and deep breathing in the sitting position, compared with those in the supine position. CONCLUSIONS: We found that the observed breathing movements were related to the effects of age, sex, and posture. These findings are in agreement with those reported in previous studies. The results may be helpful in assessing breathing movement by physical examination. Key words: breathing; breathing movement; chest wall; abdominal wall; 3-dimensional motion analysis; aging; posture; sex. [Respir Care 2012;57(9):1442-1451. () 2012 Daedalus Enterprises]
\end{abstract}

\section{Introduction}

In clinical practice, respiratory function is generally evaluated using spirometry and physical examinations. Spi-

\footnotetext{
Dr Kaneko is affiliated with the Department of Physical Therapy, School of Rehabilitation Sciences at Fukuoka, International University of Health and Welfare, Fukuoka, Japan. Dr Horie is affiliated with the Department of Physical Therapy, Faculty of Rehabilitation, Kobe International University, Hyogo, Japan.
}

This work was partly supported by grant 22500472 from the Japan Society for the Promotion of Science.

Dr Kaneko presented a version of this paper at the 16th International rometry is useful for quantitative assessments of lung volume and flow, and can be objectively compared with other spirometric results. Physical examinations, such as inspection and palpation of respiratory function, provide realCongress of the World Confederation for Physical Therapy, held June 23,
2011, in Amsterdam, The Netherlands.

The authors have disclosed no conflicts of interest.

Correspondence: Hideo Kaneko PT PhD, Department of Physical Therapy, School of Rehabilitation Sciences at Fukuoka, International University of Health and Welfare, 137-1 Enokizu, Okawa-shi, Fukuoka 8318501, Japan. E-mail: hkaneko@iuhw.ac.jp.

DOI: $10.4187 /$ respcare. 01655 
time observations and do not require a special measuring instrument; thus, they are important components of assessments in clinical settings. However, physical examinations are not quantitative and depend on the experience of the assessor. Therefore, an objective assessment of breathing movement is difficult because there are no previous reports of reference values that can be used in observational assessments of breathing movement.

The breathing movements and patterns reported by previous studies were measured by magnetometers, ${ }^{1,2}$ respiratory inductive plethysmography, ${ }^{3,4}$ and optoelectronic plethysmography..$^{5-9}$ These methods are used to estimate lung volumes from the chest and abdominal motions. The respiratory movement measuring instrument, ${ }^{10-14}$ which consists of 6 laser distance sensors, has been developed to measure changes in breathing movements of the thorax and abdomen. However, it is limited to measuring the anteroposterior diameters of breathing movements. Although several previous studies have assessed the 3-dimensional motions of the thorax and abdomen during breathing using infrared cameras ${ }^{15,16}$ and an electromagnetic device, ${ }^{17}$ there is no reported literature on the 3 -dimensional distances of the observational points on the thorax and abdomen during breathing.

Motion analysis using optical and electromagnetic devices allows accurate measurement of the kinematics of the chest and abdominal wall in different positions. An electromagnetic device requires the use of heavier sensors that are placed on the thorax and abdomen and can be affected by other devices that produce magnetic fields. On the other hand, optical devices for measuring motion require only lightweight reflective markers that cause minimum interference by the measuring system with the subjects. Recently, a study ${ }^{9}$ using an optical device showed that posture and sex strongly influenced breathing and chest wall kinematics. Therefore, we think that optical devices are more suitable for measurements of the 3-dimensional distances of observational points on the thorax and abdomen during breathing.

We believe that establishing reference values and understanding the characteristics of the breathing movements of the chest and abdominal wall are necessary for objective assessments during physical examinations. These could become the foundation for a new assessment tool for use in clinical practice. The purpose of this study was to determine the 3-dimensional distances of observational points on the thorax and abdomen during breathing in healthy subjects and to assess the effects of age, posture, and sex on breathing movements.

\section{Methods}

This study was performed at the School of Rehabilitation Sciences at Fukuoka, International University of Health

\section{QUICK LOOK}

\section{Current knowledge}

The physical assessment of breathing is important during physical therapy evaluations. The impact of posture, age, and sex on breathing movements complicates these assessments.

\section{What this paper contributes to our knowledge}

A 3-dimensional analysis of thoracic and abdominal breathing movements in healthy subjects demonstrated that patient age, sex, and posture impact both quiet and deep breathing movements. These findings may assist in future assessment in patients with pulmonary disease.

and Welfare, Fukuoka, Japan. Fifty healthy males and 50 healthy females participated in this study. Subjects were recruited from the university, hospital, and general population (students, clerks, teachers, healthcare providers, residents). We excluded those with a history of respiratory, circulatory, or neurological disorders; smoking; abnormal spirogram; respiratory symptoms; or body mass index of $>30 \mathrm{~kg} / \mathrm{m}^{2}$. The included subjects were divided into 5 age groups from 20 to 74 years of age: $20-29$ years, $30-$ 39 years, $40-49$ years, 50-59 years, and 60-74 years. Each group included 10 males and 10 females. The study was approved by the local ethics committee, and all subjects gave their informed consent.

\section{Spirometry}

Spirometry (HI-801, Chest MI, Tokyo, Japan) was performed according to American Thoracic Society standards. ${ }^{18}$

\section{Breathing Movement Measurement}

Breathing movements were measured using a 3-dimensional motion system (Vicon MX, Oxford Metrics, Oxford, United Kingdom) during quiet breathing and deep breathing in the supine and sitting positions. This system consists of 8 infrared cameras that track the movement trajectories of 14-mm passive markers attached to the thorax, chest wall, and abdominal wall. The cameras sample at a rate of $50 \mathrm{~Hz}$. Motion data were processed using the system's software (Vicon Workstation 5.2.4, Oxford Metrics, Oxford, United Kingdom) to produce 3-dimensional coordinates of each marker. The 3-dimensional coordinates were smoothed using a Woltring filter routine. The 3-dimensional distances between the markers were calculated using spreadsheet software (Excel, Microsoft, Red- 


\section{Breathing Movements of the Chest and Abdominal Wall in Healthy Subjects}

mond, Washington). We estimated the resolution to be $0.1 \mathrm{~mm}$ by measuring the signal to noise ratio while the system was recording the position of a static marker.

Thirteen passive markers were placed along the vertical line through the medial one third of the clavicle (CL), the 3rd rib (R3), the 8th rib (R8), approximately $3 \mathrm{~cm}$ below the costal margin on the lateral abdomen (LAB), along the midaxillary line on the 10th rib (R10) bilaterally, and along the vertical line through the umbilicus (the sternal angle [SA]), the xiphoid process [XP], the midpoint between the xiphoid process and umbilicus [abdomen - ABD]). In order to allow natural breathing, we designed the system so that the markers could be worn on clothing (T-shirt); $15 \times 1.2 \mathrm{~mm}$ magnets were attached to the markers and the surface of interest of the thorax and abdomen with double-faced adhesive tape.

The range of movement for each of the 2 breathing conditions was measured as the 3-dimensional distances between markers at half respiratory cycle. The distances were calculated based on the average values of 5 steady breathing cycles during quiet breathing and maximal values of 3 deep breathing movements. Additionally, respiratory rates during quiet breathing were also calculated based on the number of data analyzed. The 3-dimensional distances of each marker and the respiratory rates were calculated using spreadsheet software.

\section{Protocol}

After the anthropometric and spirometry measurements, the subjects were tested for breathing movement. Before the breathing movement measurements, the subjects were asked to wear similar T-shirts and to loosen their pants. Additionally, the female subjects were required to remove or loosen their bras. The subjects were instructed to remain as relaxed as possible on a comfortable reclining wheelchair (RR70N, Kawamura Cycle, Kobe, Japan) in the supine and sitting position, at random, and not to talk or move during the measurements. Postural change was performed by the examiner leaning the backrest of the reclining wheelchair. During quiet breathing the subjects were told to breathe as normally as possible, and the breathing movements were recorded for $1 \mathrm{~min}$. Then, during deep breathing, the subjects were asked to breathe in and out slowly up to the maximum and to repeat this pattern 3 times. The breathing movements were recorded during that time.

\section{Measurement Repeatability}

Ten healthy volunteers (mean \pm SD age $22 \pm 1$ y) were included in these measurements. All subjects were measured 3 times. Two of the measurements were performed by one examiner, and the third by another. The 2 exam- iners were physical therapy students trained in the measurement procedure. The order of measurements in each data collection session was randomized between the subjects. The subjects were examined in the supine position. During the measurements the subjects were told to rest comfortably and breathe as normally as possible. Between the measurements the subjects walked a few minutes and then returned to the supine position again. Next, the markers were placed on the defined points. Bland-Altman plotting was performed for the measurement for repeatability. The intra- and inter-rater reliabilities were analyzed using the intraclass correlation coefficient (ICC). As a result, there were no systemic differences between the 2 measurements. The ICCs $(1,1)$ were $0.66-0.94$ with standard error of measurement (SEM) values of $0.31-1.14 \mathrm{~mm}$. The ICCs $(2,1)$ were $0.63-0.95$ with SEMs of $0.34-1.19 \mathrm{~mm}$. Most of the ICCs exceeded 0.70, an acceptable level of reliability, except for the right CL (ICC $(2,1) 0.68)$, SA (ICC $(2,1) 0.69)$, and the left 10th rib marker $(\operatorname{ICC}(1,1)$ 0.66 , ICC $(2,1) 0.63)$.

\section{Statistical Analysis}

Values are expressed as mean \pm SD for age, posture, and sex groups. Paired and unpaired $t$ tests were used to compare the breathing movements between the supine and sitting position groups, and between the male and female groups, respectively. One-way analysis of variance and the Tukey multiple comparison test were used to compare the breathing movements between the age groups. To further assess the clinical importance of the breathing movement distances between the groups, an effect size coefficient $\left(\eta^{2}\right.$, Cohen's d) was calculated. Multiple regression analysis was used to assess the associations between breathing movement distance in the supine and sitting positions and age and sex after adjusting for height and weight. Statistical analyses were performed using statistics software (SPSS 14.0, SPSS, Chicago, Illinois). Values of $P<.05$ were taken to be significant.

\section{Results}

\section{Subjects}

The anthropometric and pulmonary functional data divided according to age and sex groups are summarized in Table 1. Mean height was significantly less among subjects of the 60-74-year age group than among subjects of the 20-29-year and 40-49-year age groups. FVC was significantly less in the 60-74-year-old subjects, compared to subjects of the 20-29-year, 30-39-year, and 40-49year age groups. FVC among subjects of the 50-59-year age group showed a significant decrease, compared to subjects of the 20-29-year age group. $\mathrm{FEV}_{1}$ was significantly 
Breathing Movements of the Chest and Abdominal Wall in Healthy Subjects

Table 1. Anthropometric Data for the Subjects (50 Males and 50 Females)

\begin{tabular}{|c|c|c|c|c|c|c|c|c|}
\hline Group & Age, y & $\begin{array}{l}\text { Height } \\
\text { (m) }\end{array}$ & $\begin{array}{l}\text { Weight } \\
(\mathrm{kg})\end{array}$ & $\begin{array}{c}\text { BMI } \\
\left(\mathrm{kg} / \mathrm{m}^{2}\right)\end{array}$ & $\begin{array}{l}\text { FVC } \\
(\mathrm{L})\end{array}$ & $\begin{array}{c}\mathrm{FVC} \\
(\% \text { predicted })\end{array}$ & $\begin{array}{l}\mathrm{FEV}_{1} \\
(\mathrm{~L})\end{array}$ & $\begin{array}{c}\mathrm{FEV}_{1} / \mathrm{FVC} \\
(\%)\end{array}$ \\
\hline \multicolumn{9}{|l|}{ Age, y } \\
\hline 20-29 & $21.9 \pm 1.0$ & $1.66 \pm 0.09 *$ & $58.6 \pm 9.5$ & $21.2 \pm 2.4$ & $4.43 \pm 1.05 \ddagger \S$ & $118 \pm 14$ & $4.20 \pm 0.95 \rrbracket$ & $91 \pm 5$ \\
\hline $30-39$ & $34.0 \pm 2.4$ & $1.64 \pm 0.09$ & $58.1 \pm 9.5$ & $21.6 \pm 2.3$ & $4.18 \pm 0.98 \ddagger$ & $120 \pm 16$ & $3.53 \pm 0.78^{* *}$ & $85 \pm 6$ \\
\hline $40-49$ & $44.2 \pm 2.8$ & $1.65 \pm 0.09 *$ & $59.8 \pm 13.2$ & $21.8 \pm 3.2$ & $3.96 \pm 0.88 \dagger$ & $119 \pm 17$ & $3.26 \pm 0.69 * *$ & $83 \pm 6$ \\
\hline $50-59$ & $54.6 \pm 2.9$ & $1.62 \pm 0.07$ & $59.8 \pm 9.3$ & $22.7 \pm 3.0$ & $3.53 \pm 0.75$ & $110 \pm 28$ & $2.96 \pm 0.66$ & $84 \pm 5$ \\
\hline $60-74$ & $69.4 \pm 4.3$ & $1.57 \pm 0.08$ & $56.6 \pm 6.7$ & $22.9 \pm 1.8$ & $2.92 \pm 0.69$ & $106 \pm 13$ & $2.64 \pm 0.65$ & $81 \pm 7$ \\
\hline \multicolumn{9}{|l|}{ Sex } \\
\hline Male & $44.9 \pm 17.0$ & $1.69 \pm 0.06 \|$ & $65.8 \pm 7.7 \|$ & $23.0 \pm 2.5 \|$ & $4.48 \pm 0.84 \|$ & $114 \pm 20$ & $3.78 \pm 0.91 \|$ & $83 \pm 7$ \\
\hline Female & $44.7 \pm 16.6$ & $1.56 \pm 0.06$ & $51.4 \pm 5.1$ & $21.1 \pm 2.3$ & $3.13 \pm 0.68$ & $115 \pm 18$ & $2.74 \pm 0.68$ & $86 \pm 6$ \\
\hline \multicolumn{9}{|c|}{ 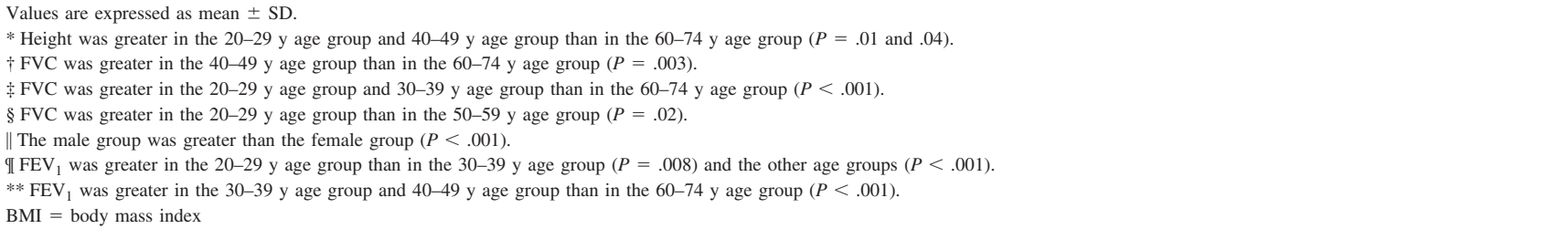 } \\
\hline
\end{tabular}

higher for the subjects of age 20-29 years than for subjects in the other age groups, and was significantly lower for the subjects of age 60-74 years than for the subjects of age 30-39 years and those age $40-49$ years. Height, weight, body mass index, and FVC were significantly greater in the males than in the females.

\section{Marker Distances}

The distances of the markers on the chest and abdomen in each age group are shown in Table 2. The average marker distances were greater for the abdomen than for the thorax in the 2 positions under the 2 breathing conditions. However, the differences in marker distances between the thorax and abdomen were smaller in the sitting position than in the supine position. In the supine position the percentage of quiet breathing marker distances relative to deep breathing marker distances ranged from $9.3 \%$ to $15.5 \%$ for the thorax and from $24.1 \%$ to $27.8 \%$ for the abdomen, whereas in the sitting position, the percentages ranged from $10.9 \%$ to $18.1 \%$ for the thorax and from $20.6 \%$ to $23.1 \%$ for the abdomen.

\section{Effect of Age}

When compared between age groups, there were no significant differences in all marker distances during quiet breathing between the 2 positions. The distances of the bilateral CL and right R3 were significantly greater during deep breathing in the supine position among subjects of the 20-29-year age group than among those of the other age groups. The SA and left R3 distances among subjects of the 20-29-year age group were significantly greater than those in subjects of the 50-59-year and 60-74-year age groups. Conversely, the XP distance in the sitting position was significantly greater in subjects of the 60 74-year age group than in subjects of the 20-29-year age group. The effect sizes $\left(\eta^{2}\right)$ for most of the markers except upper thoracic markers were small (see Table 2).

Using multiple regression analysis, during quiet breathing, there were significant negative relationships between marker distance and age for R8 in the supine position and for $\mathrm{CL}$ in the sitting position. During deep breathing there were significant negative relationships for CL, SA, and R3 in the supine position, but not in the sitting position. The model results for these markers had a moderate $\mathrm{R}^{2}$ values, ranging from 0.09 (SA during quiet breathing in the sitting position) to 0.19 (left CL during deep breathing in the supine position) (Table 3 ).

\section{Effect of Posture}

All marker distances except for the left R10 during quiet breathing and left LAB during deep breathing showed significant differences between the 2 postures, under both breathing conditions. The distances of most markers for the thorax were significantly greater in the sitting position than in the supine position, whereas all abdominal marker distances were significantly shorter during quiet breathing than during deep breathing. Conversely, the R10 distances were shorter during deep breathing in the sitting position than in the supine position. Moreover, the effect sizes (d) for more than half of the markers during quiet breathing 


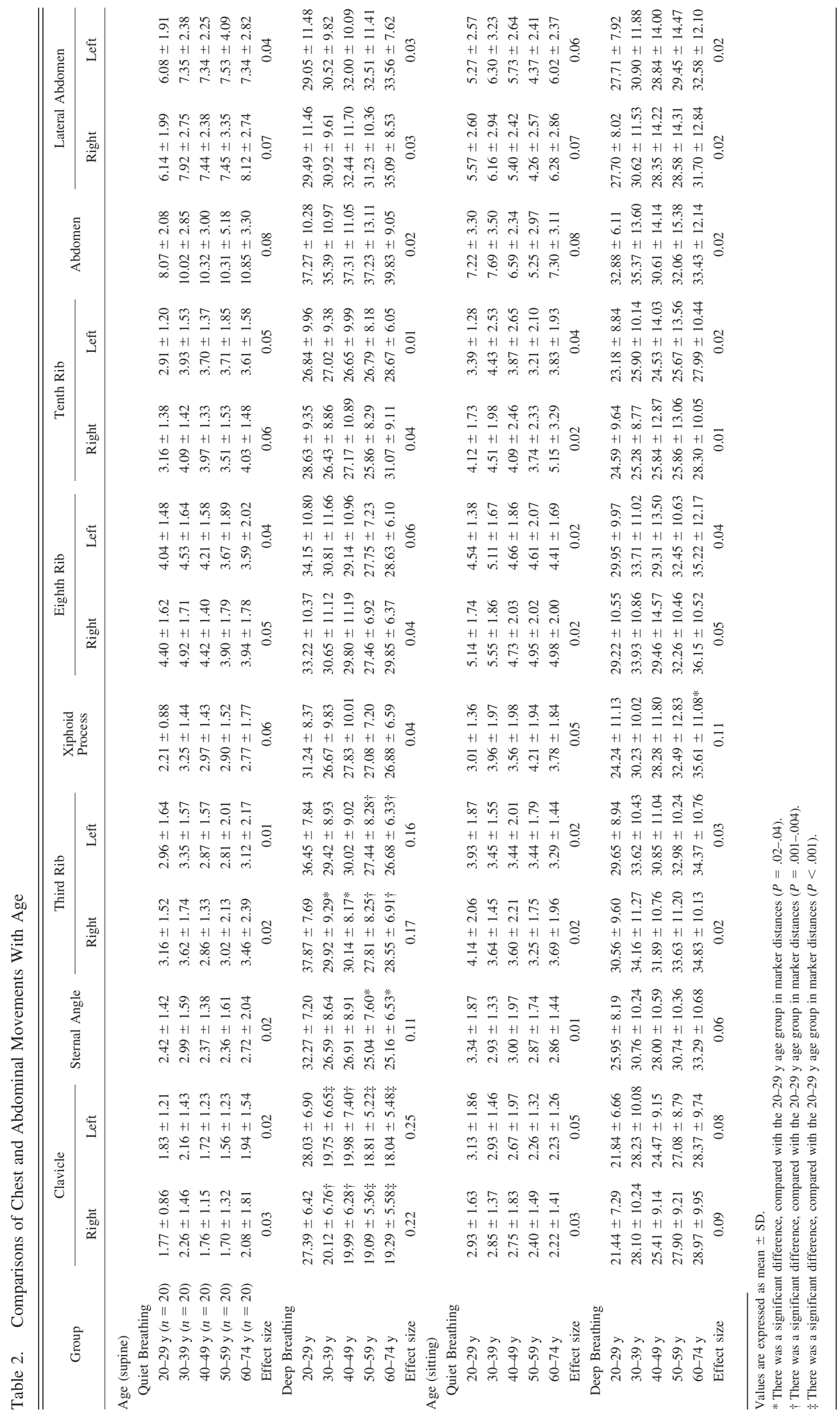




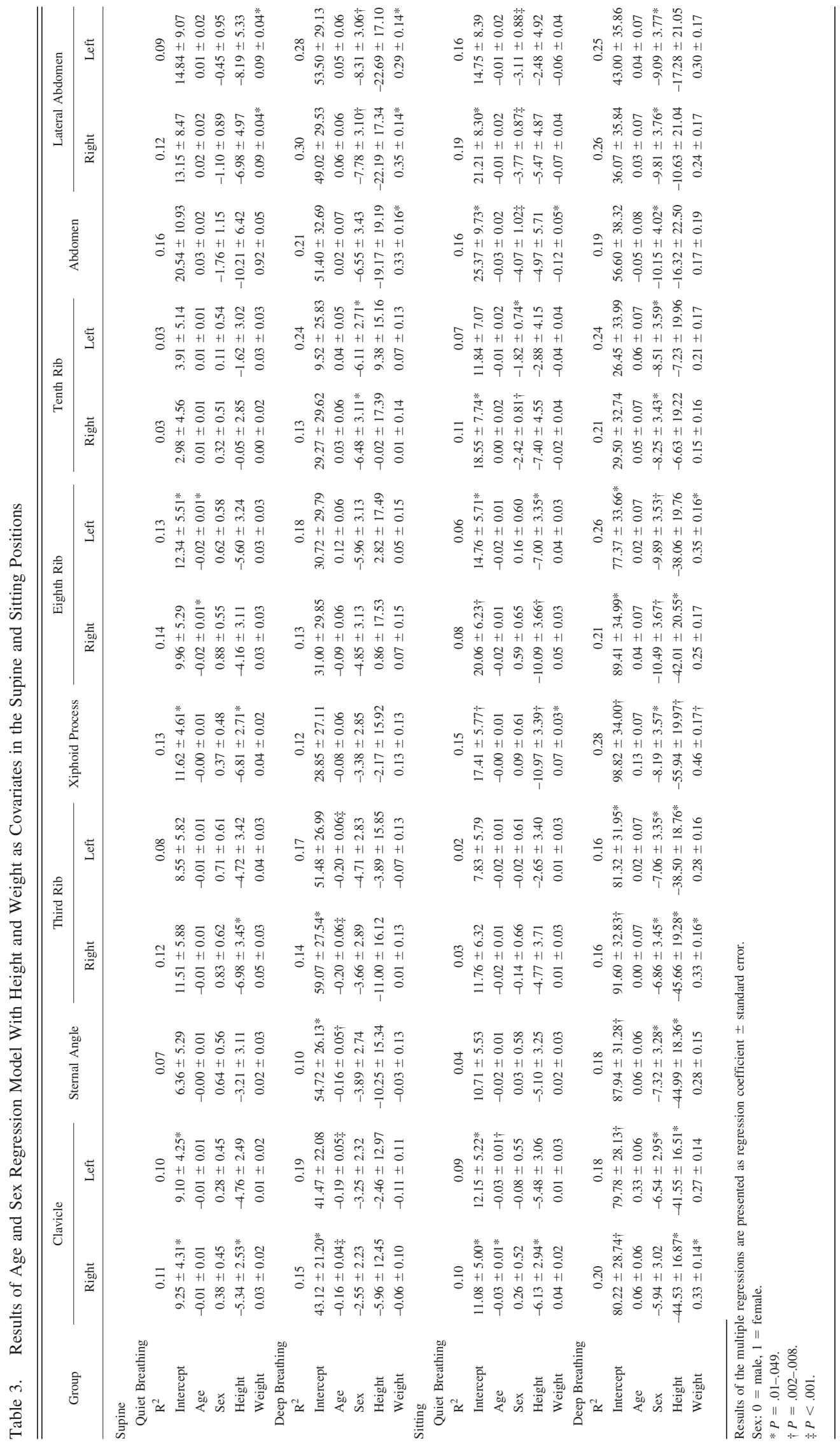




\section{Breathing Movements of the Chest and Abdominal Wall in Healthy Subjects}

were moderate, and those of for most of the markers during deep breathing were small (Table 4).

\section{Effect of Sex}

During quiet breathing in the supine position the male group had significantly shorter distances for the thoracic markers (except for R10), and significantly greater distances for the abdominal markers (except for the left LAB), compared to the female group. In the sitting position the R10 and abdominal marker distances were greater in the male group than in the female group. During deep breathing in the supine position the distances of all markers except for the CL, SA, and R3 were significantly greater in the male group than in the female group. In the sitting position there were significantly greater distances for all markers in the male group than in the female group. The effect sizes (d) for most of the markers except the upper thoracic markers during deep breathing in the supine position and during quiet breathing in the sitting position were from moderate to large (see Table 4).

By multiple regression analysis, during quiet breathing there were significant negative relationships between marker distance and sex for R10, ABD, and LAB in the sitting position, but there were no significant relationships for any of the markers in the supine position. During deep breathing there were significant negative relationships for $\mathrm{R} 10$ and $\mathrm{LAB}$ in the supine position and for all markers except for CL in the sitting position. The model results for markers with significant relationships between marker distance and sex had better than moderate $\mathrm{R}^{2}$ values, ranging from 0.09 (SA during quiet breathing in the supine position) to 0.30 (right LAB in the supine position) (see Table 3).

\section{Respiratory Rate}

Between the 2 positions during quiet breathing the mean respiratory rates were significantly higher in the sitting than in the supine position $(15.4 \pm 3.6$ breaths $/ \mathrm{min}$ and $14.6 \pm 3.7$ breaths/min, respectively). However, no significant differences were found between the sex and age groups.

\section{Discussion}

To our knowledge, this is the first study to report 3-dimensional distances of observational points on the thorax and abdomen during quiet and deep breathing. These findings confirm and expand upon previous observations regarding breathing movements. In particular, the average marker distances for the thorax and abdomen during quiet breathing were less than one third of those during deep breathing. In addition, upper thoracic movement during deep breathing and lower thoracic movement during quiet breathing in the supine position decreased with age, except during quiet breathing in the supine position; there was less abdominal movement in females than in males. These observed data should be useful for the clinical assessment of breathing movement during physical examinations, because the breathing movement that we generally observe is a 3-dimensional movement involving local regions of the chest and abdominal wall.

We measured the 3-dimensional distances of the thoracic and abdominal markers to determine reference values of breathing movements that could be used for physical examinations, such as palpation and inspection. Previous studies designed to measure specific points on the chest and abdominal walls have mainly analyzed the anteroposterior and mediolateral displacements. However, it has been reported that displacement of the ventral part of the rib cage during quiet breathing primarily occurred in the anteroposterior and craniocaudal directions. ${ }^{19}$ Leong et $\mathrm{al}^{16}$ showed that the mean displacements of thoracic and abdominal markers were greater in the craniocaudal direction than in other directions during deep breathing. We think that measuring only unidirectional displacements would be insufficient for assessments of breathing movements for various breathing patterns.

In this study, the intra- and inter-rater reliabilities of the marker distance measurements were acceptable. A previous study 20 that used the respiratory movement measuring instrument showed that the correlation for the left lower thoracic sensor was poor, whereas the correlations for the thoracic and abdominal wall sensors were moderate to strong. We found that the ICCs of the left lower thoracic markers were the lowest of all markers, which is consistent with the previous study's findings; however, the values in this study were apparently higher than those reported by Olsén and Romberg. ${ }^{20} \mathrm{We}$ assume that this difference was caused by differences in the measuring methods. In our method the 3-dimensional distances of the breathing movement markers were measured, which provided potentially more complete characterizations of the various breathing movements.

During deep breathing in the supine position the thoracic movement distances decreased with age. The results of this study are consistent with previous studies, ${ }^{4,21}$ which used different measurement devices. Verschakelen and Demedts, ${ }^{4}$ using respiratory inductive plethysmography, showed that males of $>50$ years of age in particular had shorter breathing movement distances for their rib cages. Moll and Wright ${ }^{21}$ showed, using calipers, that anteroposterior chest expansion reduced with age in normal subjects. These results appear to be attributed to age-related decreases in chest wall compliance ${ }^{22}$ and respiratory muscle strength. ${ }^{23-25}$ On the other hand, in the sitting position, while there was no significant relationship between breath- 


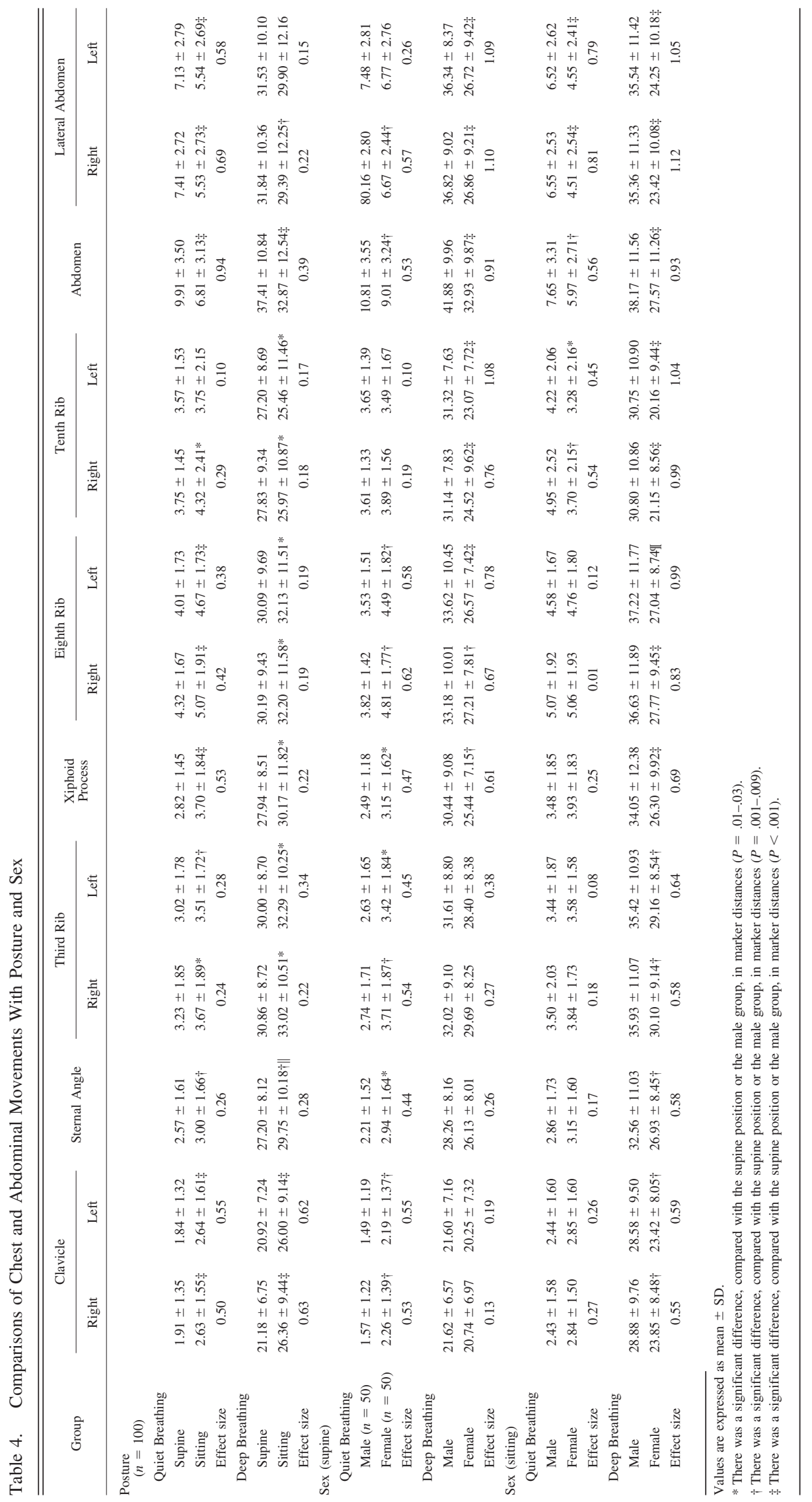




\section{Breathing Movements of the Chest and Abdominal Wall in Healthy Subjects}

ing movement and age, movements in the XP distance significantly increased in the elderly group. These results are contrary to those for the supine position. Considering that no significant differences were observed in the chest wall movement marker distances, it is possible that limited chest movement in the elderly subjects was compensated by movements that were elicited in the sitting position, for which spinal flexion movement is easier during deep breathing, even though the subjects were instructed not to move during measurement. Therefore we believe that these results were derived from limited chest movement accompanying aging.

In contrast, during quiet breathing there were no significant relationships between most of the marker distances and age, as observed in previous studies. However, R8 in the supine position and CL in the sitting position were significantly associated with age. Although we do not have a reasonable explanation for these results, we assume that these could readily be influenced by limited chest movement, which might be dependent on posture.

When compared by sex, we found that there were significantly greater thoracic marker distances and significantly shorter abdominal marker distances in the female subjects than in the male subjects during quiet breathing in the supine position. Our findings were in line with those of previous studies, $2,8,9,26,27$ which have shown that females have a wider rib cage and lower abdominal contributions to tidal volume than males during quiet breathing. However, in multiple models there were no significant relationships between breathing movement during quiet breathing in the supine position and sex. Verschakelen and Demedts $^{4}$ showed that there were no sex-related differences in thoracic and abdominal movements during quiet breathing. These discrepancies might be related to the types of measuring instruments that were used. Finally, from the present results it could be said that breathing movement during quiet breathing in the supine position is influenced by body size rather than sex, because the breathing pattern is predominantly diaphragmatic in the supine position. ${ }^{4}$

The present study also showed that during deep breathing the lower thoracic and abdominal marker distances were significantly shorter in the female subjects than in the male subjects in the supine position. Furthermore, there were also significant relationships between lower thoracic and abdominal movement and sex. Ragnarsdóttir and Kristinsdóttiri1 ${ }^{11}$ reported that abdominal, but not lower thoracic, movements were significantly less during deep breathing in females than in males. Verschakelen and Demedts $^{4}$ found that females exhibited slightly more rib cage movement during deep breathing. In other words, during deep breathing males would show predominantly diaphragmatic breathing, compared to females. The diaphragm has a mechanism of expansion that involves not only the abdomen but also the lower thorax. ${ }^{28}$ Therefore we assumed that the marker distances of the lower thorax were greater in the male subjects. In the sitting position, female subjects had significantly decreased upper thoracic movement, and there were significant negative relationships between upper thoracic movement and sex. Although the reason for this is not clear, it is possible that the thoracic movement for the male was overestimated by adding spinal flexion movement, because the increase in average thoracic marker distance was greater in male subjects than in female subjects. Thus, we believe that there were no sex-related differences in upper thoracic movement.

This study showed that posture had an influence on breathing movements during quiet breathing. This finding is consistent with those of previous studies that have shown that the contribution of the rib cage to tidal volume was greater in the sitting position than in the supine position. 1,4,9,26 This effect has been explained by the decreased compliance of the rib cage and increased compliance of the diaphragm and abdomen that results with the change in position from sitting to supine. ${ }^{22,29}$ During deep breathing the results we observed are in line with those of a previous study reported by Verschakelen and Demedts, ${ }^{4}$ indicating that the rib cage dominance in breathing patterns was greater during deep breathing than during quiet breathing. However, the changes in the R10 marker distances between the 2 positions were opposite to those observed during quiet breathing. The cause of this difference is not known. Possible causes are more predominant rib cage breathing and higher lung volumes. Loring and $\mathrm{Mead}^{28}$ showed that the inspiratory action of the diaphragm on the rib cage was greatest at low lung volumes. Accordingly, during deep breathing, which involves higher lung volumes and relatively greater rib cage movement, expansion of the lower rib cage seems to be more difficult in the sitting position than in the supine position.

As described above, most of our findings regarding the effects of age, sex, and posture are in agreement with those of previous studies. We found that the 3-dimensional distances of the thoracic and abdominal markers can represent the variable features of breathing movements. Therefore we assume that the observed data should be useful for observational assessments of breathing movement during physical examinations, and for which it will be necessary to develop a new clinical assessment tool that specifies positions, observation point, graded scaling, and other factors. However, the sample size in this study was relatively small and the individual variability in the breathing movements was large. Given that the observed values in the present study were not considerably different from the range of previously presented values, they might be used as reference values in assessing breathing movements. Moreover, in this study the tidal volume during quiet breathing was not measured, in order to avoid unnatural breathing. We therefore cannot discuss the effect of tidal volume 


\section{Breathing Movements of the Chest and Abdominal Wall in Healthy Subjects}

on breathing movement. In previous studies there were no significant effects of age and posture on tidal volume, and of sex on normalized tidal volume, and there were no significant differences in respiratory rates. ${ }^{9,25}$ Considering that respiratory rates were significantly higher in the sitting position than in the supine position, it is possible that the tidal volume was lower when sitting than when supine, assuming that minute ventilation was the same between the 2 positions. However, we believe that there was little effect on tidal volume because the differences in respiratory rates were small.

\section{Conclusions}

In this study, we measured the 3-dimensional distances of the thoracic and abdominal breathing movements in healthy subjects for use in future observational assessments of breathing movement. We found that the observed breathing movements were related to the effects of age, sex, and posture, which are findings shown in previous studies. The results in this study may be helpful in assessments of breathing movement by physical examination.

\section{REFERENCES}

1. Sharp JT, Goldberg NB, Druz WS, Danon J. Relative contributions of rib cage and abdomen to breathing in normal subjects. J Appl Physiol 1975;39(4):608-618.

2. Gilbert R, Auchincloss JH Jr, Peppi D. Relationship of rib cage and abdomen motion to diaphragm function during quiet breathing. Chest 1981;80(5):607-612.

3. Tobin MJ, Chadha TS, Jenouri G, Birch SJ, Gazeroglu HB, Sackner MA. Breathing patterns. 1. Normal subjects. Chest 1983;84(2):202-205.

4. Verschakelen JA, Demedts MG. Normal thoracoabdominal motions. Influence of sex, age, posture, and breath size. Am J Respir Crit Care Med 1995;151(2 Pt 1):399-405.

5. Cala SJ, Kenyon CM, Ferrigno G, Carnevali P, Aliverti A, Pedotti A, et al. Chest wall and lung volume estimation by optical reflectance motion analysis. J Appl Physiol 1996;81(6):2680-2689.

6. Aliverti A, Dellaca R, Pelosi P, Chiumello D, Pedotti A, Gattinoni L. Optoelectronic plethysmography in intensive care patients. Am J Respir Crit Care Med 2000;161(5):1546-1552.

7. Aliverti A, Dellaca R, Pelosi P, Chiumello D, Gatihnoni L, Pedoti A. Compartmental analysis of breathing in the supine and prone positions by optoelectronic plethysmography. Ann Biomed Eng 2001; 29(1):60-70

8. Binazzi B, Lanini B, Bianchi R, Romagnoli I, Nerini M, Gigliotti F, et al. Breathing pattern and kinematics in normal subjects during speech, singing and loud whispering. Acta Physiol 2006;186(3):233-246.

9. Romei M, Mauro AL, D'Angelo MG, Turconi AC, Bresolin N, Pedotti A, et al. Effects of gender and posture on thoraco-abdominal kinematics during quiet breathing in healthy adults. Respir Physiol Neurobiol 2010;172(3):184-191.
10. Ragnarsdóttir M, Kristjansdóttir A, Ingvarsdóttir I, Hannesson $\mathrm{P}$, Torfason B, Cahalin L. Short-term changes in pulmonary function and respiratory movements after cardiac surgery via median sternotomy. Scand Cardiovasc J 2004;38(1):46-52.

11. Ragnarsdóttir M, Kristinsdóttir EK. Breathing movements and breathing patterns among healthy men and women 20-69 years of age. Reference values. Respiration 2006;73(1):48-54.

12. Ragnarsdóttir M, Geirsson AJ, Gudbjornsson B. Rib cage motion in ankylosing spondylitis patients: a pilot study. Spine J 2008;8(3):505509.

13. Kristjansdóttir A, Ragnarsdóttir M, Hannesson P, Beck HJ, Torfason B. Chest wall motion and pulmonary function are more diminished following cardiac surgery when the internal mammary artery retractor is used. Scand Cardiovasc J 2004;38(6):369-374.

14. Kristjansdóttir A, Ragnarsdóttir M, Hannesson P, Beck HJ, Torfason B. Respiratory movements are altered three months and one year following cardiac surgery. Scand Cardiovasc J 2004;38(2):98-103.

15. Ferrigno G, Carnevali P, Aliverti A, Molteni F, Beulcke G, Pedotti A. Three-dimensional optical analysis of chest wall motion. J Appl Physiol 1994;77(3):1224-1231.

16. Leong JC, Lu WW, Luk KD, Karlberg EM. Kinematics of the chest cage and spine during breathing in healthy individuals and in patients with adolescent idiopathic scoliosis. Spine 1999;24(13):1310-1315.

17. Culham EG, Jimenez HA, King CE. Thoracic kyphosis, rib mobility, and lung volumes in normal women and women with osteoporosis. Spine 1994;19(11):1250-1255.

18. American Thoracic Society. Standardization of spirometry-1994 update. Am J Respir Crit Care Med 1995;152(3):1107-1136.

19. De Groote A, Wantier M, Cheron G, Estenne M, Paiva M. Chest wall motion during tidal breathing. J Appl Physiol 1997;83(5):1531-1537.

20. Olsén MF, Romberg K. Reliability of the Respiratory Movement Measuring Instrument, RMMI. Clin Physiol Funct Imaging 2010; 30(5):349-353

21. Moll JM, Wright V. An objective clinical study of chest expansion. Ann Rheum Dis 1972;31(1):1-8.

22. Estenne M, Yernault JC, De Troyer A. Rib cage and diaphragmabdomen compliance in humans: effects of age and posture. J Appl Physiol 1985;59(6):1842-1848.

23. Enright PL, Adams AB, Boyle PJ, Sherrill DL. Spirometry and maximal respiratory pressure references from healthy Minnesota 65- to 85-year-old women and men. Chest 1995;108(3):663-669.

24. Wijkstra PJ, van der Mark TW, Boezen M, van Altena R, Postma DS, Koeter GH. Peak inspiratory mouth pressure in healthy subjects and in patients with COPD. Chest 1995;107(3):652-656.

25. Britto RR, Zampa CC, de Oliveira TA, Prado LF, Parreira VF. Effects of the aging process on respiratory function. Gerontology 2009;55(5):505-510.

26. Fugl-Meyer AR. Relative respiratory contribution of the rib cage and the abdomen in males and females with special regard to posture. Respiration 1974;31(3):240-251.

27. Bellemare F, Jeanneret A, Couture J. Sex differences in thoracic dimensions and configuration. Am J Respir Crit Care Med 2003; 168(3):305-312.

28. Loring SH, Mead J. Action of the diaphragm on the rib cage inferred from a force-balance analysis. J Appl Physiol 1982;53(3):756-760.

29. Konno K, Mead J. Static volume-pressure characteristics of the rib cage and abdomen. J Appl Physiol 1968;24(4):544-548. 